\title{
ASOCIACIÓN ENTRE LACTANCIA MATERNA EXCLUSIVA Y DERMATITIS ATÓPICA EN UN HOSPITAL DE TERCER NIVEL DE ATENCIÓN
}

\author{
Winston Maldonado-Gómez ${ }^{1,2, a, b}$, Janet Chuan-Ibáñez ${ }^{1, c}$, Génesis Guevara-Vásquez 1,d ${ }^{\text {, César Gutiérrez }}{ }^{3, e, f}$, \\ Jorge Sosa-Flores ${ }^{2, c, g}$
}

\begin{abstract}
RESUMEN
Objetivos. Evaluar si la lactancia materna exclusiva se asocia a la dermatitis atópica en niños menores de siete años. Materiales y métodos. Estudio tipo casos y controles no pareado, realizado en un hospital de tercer nivel de atención (Hospital Regional Lambayeque) entre junio de 2016 y mayo de 2018 en donde se evaluó 260 casos y 260 controles captados en consulta externa de los servicios de Dermatología y Pediatría. Se usaron los criterios del Reino Unido para el diagnóstico de dermatitis atópica y una encuesta con posibles factores de riesgo. Resultados. En el análisis bivariado se encontró diferencias entre los casos y controles en relación a lactancia materna exclusiva $(p=0,001)$, antecedente familiar de atopia $(p<0,001)$, nivel educativo de padres $(p<0,001)$, hábito de fumar en presencia del niño $(p=0,006)$ y procedencia $(p=0,002)$. La lactancia materna exclusiva estuvo presente en $93(35,8 \%)$ de los casos en comparación con $132(50,8 \%)$ en los controles. En el análisis multivariado usando regresión logística se encontró que lactancia materna exclusiva tendría un efecto protector sobre la presencia de dermatitis atópica $(O R=0,62 ; \mathrm{IC} 95 \%=0,41-0,94)$ ajustado por edad, sexo, procedencia, nivel educativo de los padres, inicio de alimentación complementaria, familiar con atopía y hábito de fumar. Conclusiones. La lactancia materna materna exclusiva tendría un efecto protector sobre la presencia de dermatitis atópica en niños menores de siete años.
\end{abstract}

Palabras claves: Dermatitis atópica; Lactancia materna; Niño (fuente: DeCS BIREME).

\section{ASSOCIATION BETWEEN EXCLUSIVE BREASTFEEDING AND ATOPIC DERMATITIS IN A THIRD LEVEL OF CARE HOSPITAL}

\begin{abstract}
Objetive. To assess whether exclusive breastfeeding is associated with atopic dermatitis in children under seven years of age. Materials and Methods. Non-paired case and control type study conducted in a third level of care hospital (Lambayeque Regional Hospital) between June 2016 and May 2018 in which 260 cases and 260 controls recruited in outpatient consultation of the Dermatology and Pediatrics services were evaluated. The UK criteria were used for the diagnosis of atopic dermatitis, as well as a survey with possible risk factors. Results. In the bivariate analysis, differences were found between cases and controls in relation to exclusive breastfeeding $(p=0.001)$, family history of atopy $(p<0.001)$, parents' educational level $(p<0.001)$, smoking in the presence of the child $(p=0.006)$, and origin $(p=0.002)$. Exclusive breastfeeding was present in $93(35.8 \%)$ of cases compared to $132(50.8 \%)$ of controls. The multivariate analysis using logistic regression found that exclusive breastfeeding would have a protective effect on the presence of atopic dermatitis $(\mathrm{OR}=0.62 ; 95 \% \mathrm{Cl}=0.41-0.94)$ adjusted by age, sex, background, parents' educational level, initiation of complementary feeding, family member with atopy, and smoking habit. Conclusions. Exclusive breastfeeding would have a protective effect on the appearance of atopic dermatitis in children under the age of seven.
\end{abstract}

Keywords: Atopic dermatitis, Breastfeeding, infant (source: MeSH NLM).

\footnotetext{
Hospital Regional Lambayeque. Chiclayo, Perú.

Facultad de Medicina Humana, Universidad Nacional Pedro Ruiz Gallo. Lambayeque, Perú.

Facultad de Medicina Universidad de Piura. Lima, Perú.

Médico dermatólogo; ${ }^{\mathrm{b}}$ maestro en Medicina; ${ }^{\mathrm{c}}$ médico pediatra; ${ }^{\mathrm{d}}$ licenciada estadística; ${ }^{\mathrm{e}}$ médico cirujano; ${ }^{\mathrm{f}}$ magister en Epidemiología; ${ }^{\mathrm{g}}$ doctor en Ciencias.

Este artículo forma parte de la tesis «Asociación entre lactancia materna exclusiva y dermatitis atópica en consulta externa de un hospital de tercer nivel de atención» para obtener el grado de maestro en Medicina de Winston Maldonado-Gómez por la Universidad Nacional Pedro Ruiz Gallo 2018. Disponible en: http:// repositorio.unprg.edu.pe/handle/UNPRG/2558

Recibido: 09/01/2019 Aprobado: 22/05/2019 En línea: 28/06/2019
}

Citar como: Maldonado-Gómez W, Chuan-Ibáñez J, Guevara-Vásquez G, Gutiérrez C, Sosa-Flores J. Asociación entre lactancia materna exclusiva y dermatitis atópica en un hospital de tercer nivel de atención. Rev Peru Med Exp Salud Pública. 2019;36(2):239-46. doi: http://dx.doi.org/10.17843/rpmesp.2019.362.4180. 


\section{INTRODUCCIÓN}

La Dermatitis Atópica (DA) es una enfermedad inflamatoria crónica cutánea de la infancia recurrente; asociada a defectos de barrera en la piel que la hacen susceptible a alérgenos e irritantes ${ }^{(1)}$. Su prevalencia se ha incrementado según lo encontrado en una revisión sistemática realizada de estudios publicados entre 1990 y $2010{ }^{(2)}$. A nivel de Latinoamérica el International Study of Asthma and Allergies in Childhood (ISAAC) fase tres reportó el 2010 una prevalencia de $11,3 \%$ en niños de seis a siete años, y una prevalencia de $12,8 \%$ en adolescentes de Lima ${ }^{(3)}$. El tratamiento de dermatitis atópica genera costos elevados tanto para la familia como para el estado ${ }^{(4)}$. Asimismo presenta un mayor impacto en la calidad de vida asociado a una mayor severidad del prurito ${ }^{(5)}$

Los factores asociados a la DA son: un tamaño familiar pequeño, mayores ingresos económicos en la familia, un mayor nivel educativo de los padres, el vivir en áreas urbanas; y antecedentes familiares de atopía, especialmente el materno ${ }^{(1)}$.

El diagnóstico de DA se hace clínicamente basado en antecedentes, morfología y distribución de las lesiones cutáneas y signos clínicos asociados, Willians et al. conformaron el Grupo de Trabajo del Reino Unido para el diagnóstico de DA; siendo validados estos criterios a nivel hospitalario, presentando una sensibilidad de $85 \%$ y una especificidad de $96 \%{ }^{(6,7)}$. Dharma et al. en un estudio realizado en Canadá encuentran que los datos obtenidos por un cuestionario no pueden substituir la evaluación por un profesional de salud usando criterios diagnósticos clínicos validados ${ }^{(8)}$.

Diversos estudios muestran contradicciones sobre la asociación entre la lactancia materna y la dermatitis atópica, algunos encuentran un efecto protector de la lactancia materna y otros encuentran que sería un factor de riesgo, estas controversias se deberían a diferencias en los diseños de estos trabajos y diferencias en la forma de medir las variables. Algunos definen DA a través de la aplicación de un cuestionario sobre antecedentes de diagnóstico médico, otros utilizan la evaluación médica para hacer el diagnóstico; asimismo son diferentes la forma de medir la lactancia materna: duración de lactancia materna, lactancia materna exclusiva (LME) o sólo el haber recibido lactancia materna; además, la mayoría de los trabajos, provienen de Europa y Asia ${ }^{(9-25)}$, por tanto, se plantea realizar un trabajo para evaluar esta asociación en nuestro medio.

El objetivo del estudio fue determinar si la LME se asocia a la protección contra el desarrollo de DA en niños menores de

\section{MENSAJES CLAVE}

Motivación para realizar el estudio. Algunos estudios describen que la lactancia materna exclusiva tendría un efecto protector en el desarrollo de la dermatitis atópica, sin embargo, otros estudios refieren que seria un factor de riesgo. En Perú no se ha encontrado estudios que evalúen dicha asociación.

Principales hallazgos. Encontramos un efecto protector de la lactancia materna en relación a la dermatitis atópica, la bibliografía explica que esto sucede en los casos más severos de dermatitis atópica.

Implicancias. Sería necesario realizar trabajos que confirmen estos hallazgos, a fin de poder implementar la lactancia materna exclusiva como una medida de prevención de la dermatitis atópica, con impacto en la calidad de vida infantil.

siete años atendidos en consulta externa de dermatología y pediatría de un hospital de tercer nivel de atención de Perú.

\section{MATERIALES Y MÉTODOS}

\section{DISEÑO DEL ESTUDIO Y POBLACIÓN}

Se realizó un estudio analítico, retrospectivo, con diseño de tipo casos y controles no pareado en niños menores de siete años atendidos en la consulta externa de los servicios de dermatología y pediatría del Hospital Regional Lambayeque entre junio de 2016 y mayo de 2018. El Hospital Regional Lambayeque es un hospital de tercer nivel de atención ubicado en la ciudad de Chiclayo, región Lambayeque, el cual atiende a población perteneciente a la macrorregión norte del Perú.

\section{MUESTRA Y MUESTREO}

Se calculó la muestra para un diseño de tipo casos y controles con el paquete estadístico Epiinfo versión 7, considerando un nivel de confianza del $95 \%$, una potencia del $80 \%$, un valor de odds ratio (OR) probable de 0,6 tomando en cuenta un valor intermedio entre lo reportado por Goicochea et al. $(0,53){ }^{(25)}$ y otros antecedentes encontrados. Además de una frecuencia de lactancia materna exclusiva en los controles de $68,4 \%$. ${ }^{(26)}$. Logrando obtener finalmente 260 casos y 260 controles, los cuales fueron encuestados de manera consecutiva ya que se utilizó el muestreo no probabilístico consecutivo.

Para la selección de los casos se tomaron como criterios de inclusión: a) pacientes con una edad < 7 años; y b) pacientes con diagnóstico de DA determinado mediante el uso de los criterios diagnósticos del Grupo de Trabajo del Reino Unido. Asimismo, se consideraron los siguientes criterios de exclusión: a) pacientes con alguna contraindicación para recibir lactancia materna exclusiva, por ejemplo, 
tener madre con diagnóstico de infección por Virus de Inmunodeficiencia Humana ( $\mathrm{VIH}$ ) y b) niños que presenten cuadro clínico que corresponde al diagnóstico diferencial de DA como escabiosis, dermatitis seborreica y dermatitis de contacto.

Para la selección de los controles se tomaron como criterios de inclusión: a) pacientes varones y mujeres con una edad <7 años; y b) pacientes varones y mujeres que no cumplieron con los criterios diagnósticos de DA tras la evaluación por un médico dermatólogo o pediatra. Se excluyeron: a) niños con alguna contrindicación para presentar lactancia materna exclusiva como madre con diagnóstico infección por $\mathrm{VIH}$, b) pacientes con evidencia clínica o diagnóstico ya realizado de asma bronquial, dermatitis atópica o rinitis alérgica; y c) pacientes varones y mujeres que no tuvieron la cartilla de control de crecimiento y desarrollo otorgada por el Ministerio de Salud, o en los cuales no se tuvo accesibilidad a su historia clínica neonatal.

\section{VARIABLE DEPENDIENTE}

El diagnóstico de DA fue determinada por el médico tratante (dermatólogo o pediatra) en consultorios según criterios diagnósticos del Grupo de Trabajo del Reino Unido; es decir, un niño tenía DA si presentaba prurito y al menos tres de los siguientes criterios: historia de dermatitis flexural, historia personal o familiar de atopia, historia de piel seca en el último año, dermatitis flexural visible y el inicio de la enfermedad antes de los dos años. Estos criterios diagnóstico fueron validados a nivel hospitalario, presentando una sensibilidad de $85 \%$ y una especificidad de $96 \%{ }^{(6)}$.

\section{VARIABLES INDEPENDIENTES}

Las variables en estudio fueron obtenidas a través de una encuesta no autoadministrada, el sexo y la edad del niño fueron tomadas de la tarjeta de identificación. La LME hasta los seis meses de edad fue definida como lactancia materna sin recibir otro tipo de alimento o suplemento, para lo cuál se preguntó sobre la duración en meses de la lactancia, si hubo lactancia mixta y su edad de inicio. El inicio de alimentación complementaria fue considerado como adecuada si ocurrió a los seis meses o más, en caso contrario fue definido como temprano. Para controlar el probable sesgo de medición en esta variable (Inicio de alimentación complementaria) se incluyó una pregunta referente al inicio de consumo de alimentos, además de la lactancia materna ${ }^{(25)}$. El nivel educativo se definió según el máximo nivel educativo alcanzado de alguno de los padres, clasificándose como básica y superior. La zona de procedencia, se definiò de acuerdo a la procedencia de la vivienda, como urbana y rural. El antecedente de atopia por familiar directo fue considerado presente si el padre, la madre y/o hermanos fueron diagnósticados alguna vez y/o recibieron tratamiento para rinitis alérgica, asma bronquial o dermatitis atópica. Finalmente el hábito de fumar en presencia del niño se consideró por el trabajo de Morales Suárez-Valera sobre el hábito de fumar y la prevalencia de DA ${ }^{(27)}$; es decir, si en alguna oportunidad hubo exposición pasiva por alguno de los miembros directos de la familia.

Todas las variables fueron medidas sin considerar la temporalidad de presentación de cada factor y del evento.

\section{PROCEDIMIENTOS}

La recolección de datos fue realizada en consultorios externos de pediatría y dermatología del Hospital Regional Lambayeque (III-1), según turno correspondiente, por los médicos especialistas para tratar de excluir los diagnósticos diferenciales de DA. Una vez identificado al potencial participante y después de terminada su atención, el padre o tutor firmó el consentimiento informado previa explicación y aceptación; posteriormente un personal de apoyo, el cual fue capacitado, realizó el llenado de la ficha de recolección en un ambiente paralelo al consultorio y no fue autoadministrada. La ficha fue elaborada para tal fin y cuenta con 12 preguntas puntuales, por lo que no se realizó piloto para su adecuación. El tiempo de llenado fue de aproximadamente de cinco a diez minutos.

\section{ANÁLISIS ESTADÍSTICO}

Antes de comenzar con el análisis descriptivo, se corroboró la distribución de la muestra en estudio con la prueba de normalidad de Kolmogorov-Smirnov, considerando un nivel de significancia del $5 \%$. Posterior a ello, las variables cuantitativas fueron presentadas en medidas de tendencia central y de dispersión; es decir, fueron presentadas en medianas y rangos intercuartílicos; y las variables cualitativas, se resumieron en frecuencias absolutas y relativas (porcentajes). El análisis bivariado fue realizado con la prueba Chi cuadrado y prueba $U$ de Mann Whitney, a fin de evidenciar las diferencias entre casos y controles, considerando significativos aquellos con valor de $p<0,05$.

Para evaluar la asociación entre la LME y la DA, se calculó el odds ratio (OR) crudo y ajustado por las variables en estudio, estos fueron presentados con intervalos de confianza al 95\% mediante el modelo de Regresión Logística con la metodología Stepwise. En el análisis crudo se presentan los valores de $\mathrm{p}$ al $5 \%$ de significancia y los OR de forma independiente. En el análisis multivariado ingresaron todas las variables significativas (Procedencia, nivel educativo máximo alcanzado de los padres, antecedente de atopia por familiar directo, hábito de fumar en presencia del niño) y aquellas con significancia clínica que se encuentran asociadas a la variable dependiente (edad, sexo y alimentación complementaria), ambas fueron consideradas en el modelo ajustado final.

Se utilizó con un nivel de significancia del $5 \%$ para el test de bondad de ajuste de Hosmer- Lemeshow con la finalidad de evidenciar un buen ajuste entre las probabilidades 
estimadas y los valores observados de las variables establecidas en el modelo final; es decir, no ajenas de la realidad.

Los datos recolectados en las fichas fueron ingresados al programa MS Excel versión 2010, con el objetivo de verificar la existencia datos faltantes en alguna de las preguntas, sin embargo no se encontraron datos perdidos o vacíos, por lo que no se eliminaron casos y tampoco se utilizaron métodos de imputación de datos. El análisis correspondiente fue realizado con el programa STATA versión 14 (Stata Corporation, College Station, Texas, USA).

\section{CONSIDERACIONES ÉTICAS}

Este trabajo fue revisado y aprobado por el Comité de Ética en Investigación del Hospital Regional Lambayeque. La participación en el estudio se realizó previo llenado de la ficha del consentimiento informado. La información se almacenó en forma codificada, evitando toda información que permitiera la identificación de las personas. La información sólo fue usada para los propósitos de la investigación.

\section{RESULTADOS}

Con relación a la muestra se obtuvieron datos de 260 casos y 260 controles cuyos padres o apoderados fueron entrevistados en consultorio externo de dermatología y pediatria del Hospital Regional Lambayeque entre mayo de 2016 y abril de 2018.

Entre las características generales de la muestra se observa que la mediana de la edad fue de 29,1 meses, con un rango intercuartílico (P25-P75) de 14,2 a 55,4 meses, el sexo fue femenino en el $50,4 \%$ y la lactancia fue exclusiva en el $43,3 \%$; tuvieron antecedente familiar de alergia el 53,3\% y el nivel educativo de los padres fue nivel superior en $63,1 \%$; iniciaron alimentación complementaria en forma adecuada el 86,5\%; el antecedente de hábito de fumar en casa por algunos de sus parientes directos estuvo presente en el $14,2 \%$ y la procedencia fue urbana en el $89,2 \%$. Cuando se realiza el análisis bivariado entre casos y controles se observa que la LME estuvo presente en 93 (35,8\%) de los casos en comparación con $132(50,8 \%)$ en los controles. (Tabla 1).

Tabla 1. Características de los niños con y sin dermatitis atópica atendidos en un hospital de tercer nivel, 2016 - 2018

\begin{tabular}{|c|c|c|c|c|c|c|c|}
\hline \multirow{2}{*}{ Características } & \multirow{2}{*}{$\mathbf{N}$} & \multirow{2}{*}{$\%$} & \multicolumn{2}{|c|}{ Controles } & \multicolumn{2}{|c|}{ Casos } & \multirow{2}{*}{ Valor de $\mathrm{p}$} \\
\hline & & & $\mathbf{n}$ & $\%$ & $\mathrm{n}$ & $\%$ & \\
\hline Lactancia materna exclusiva & & & & & & & 0,001 \\
\hline No & 295 & 56,7 & 128 & 49,2 & 167 & 64,2 & \\
\hline Sí & 225 & 43,3 & 132 & 50,8 & 93 & 35,8 & \\
\hline Mediana de edad del niño en meses (RIC) & $29,1(14,2-$ & $55,4)$ & $32,3(14,0$ & $-56,8)$ & $27,8(14,4-$ & $-53,6)$ & 0,397 \\
\hline Sexo & & & & & & & 0,114 \\
\hline Masculino & 258 & 49,6 & 138 & 53,1 & 120 & 46,2 & \\
\hline Femenino & 262 & 50,4 & 122 & 46,9 & 140 & 53,9 & \\
\hline Procedencia & & & & & & & 0,002 \\
\hline Rural & 56 & 10,8 & 39 & 15,0 & 17 & 6,5 & \\
\hline Urbano & 464 & 89,2 & 221 & 85,0 & 243 & 93,5 & \\
\hline Nivel educativo máximo alcanzado de los padres & & & & & & & $<0,001$ \\
\hline Básica & 192 & 36,9 & 131 & 50,4 & 61 & 23,5 & \\
\hline Superior & 328 & 63,1 & 129 & 49,6 & 199 & 76,5 & \\
\hline Inicio de alimentación complementaria & & & & & & & 0,304 \\
\hline Temprana & 70 & 13,5 & 39 & 15,0 & 31 & 11,9 & \\
\hline Adecuada & 450 & 86,5 & 221 & 85,0 & 229 & 88,1 & \\
\hline Antecedente de atopia de familiar directo & & & & & & & $<0,001$ \\
\hline No & 243 & 46,7 & 171 & 65,8 & 72 & 27,7 & \\
\hline Sí & 277 & 53,3 & 89 & 34,2 & 188 & 72,3 & \\
\hline Hábito de fumar en presencia del niño & & & & & & & 0,006 \\
\hline No & 446 & 85,8 & 234 & 90,0 & 212 & 81,5 & \\
\hline Sí & 74 & 14,2 & 26 & 10,0 & 48 & 18,5 & \\
\hline
\end{tabular}

${ }^{*}$ RIC: rango intercuartil 
En el análisis de asociación cruda se encontró valores de $p$ significativos para lactancia materna exclusiva $(p=0,001)$, antecedente de atopía $(p<0,001)$, nivel educativo de los padres $(p<0,001)$, hábito de fumar en la casa $(p=0,006)$, procedencia $(p=0,002)$; no habiendo diferencia estadísticamente significativa para edad $(p=0,253)$, sexo $(p=0,115)$ e inicio de alimentación complementaria $(p=0,305)$. Con relación al OR para LME en la prueba de regresión logística se encontró que los niños que tuvieron LME presentaron $38 \%$ menos riesgo de desarrollar DA en comparación con aquellos niños que no tuvieron LME ajustado por antecedente de atopia por familiar directo, nivel educativo máximo alcanzado de los padres, hábito de fumar en presencia del niño, la edad, el sexo, inicio de alimentación complementaria y procedencia $(\mathrm{OR}=0,62$; $95 \%$ IC $=0,41-0,94$ ) (Tabla 2). Cuando se aplicó la prueba de bondad de ajuste de Hosmer Lemeshow se obtuvo un valor de chi cuadrado de $8,05(p=0,430)$.

\section{DISCUSIÓN}

En el presente estudio la LME tuvo un efecto protector para DA ajustado por antecedentes de atopía, el ingreso de alimentación complementaria, sexo, edad y el hábito de fumar en presencia del niño; hallazgos que concuerdan con lo reportado en estudios previos referidos al tema ${ }^{(11,13,18,19)}$. Sin embargo, nuestros resultados no concuerdan con lo observado en un estudio realizado en niños de escuelas primarias en México ${ }^{(20)}$, lo cual podría explicarse por la distinta población evaluada.

Asimismo, Bjorkstein et al. ${ }^{(18)}$ en concordancia con lo encontrado en el presente estudio, en un estudio transversal en el cual usa los datos de la fase III del estudio ISAAC en 72 centros de 31 países en niños de seis a siete años, encuentran que la LME se asocia a una menor frecuencia de DA severa, por tanto a pesar de las limitaciones que presenta un estudio transversal, el hecho de haber incluido una muestra muy amplia y de diversas partes del mundo

Tabla 2. Asociación entre dermatitis atópica y lactancia materna exclusiva en niños atendidos en un hospital de tercer nivel, 2016-2018

\begin{tabular}{|c|c|c|c|c|c|c|}
\hline \multirow{2}{*}{ Características } & \multicolumn{3}{|c|}{ Crudo } & \multicolumn{3}{|c|}{ Ajustado* } \\
\hline & OR & IC $95 \%$ & Valor de $p$ & OR & IC $95 \%$ & Valor de $p$ \\
\hline \multicolumn{7}{|c|}{ Lactancia materna exclusiva } \\
\hline No & 1 & & & 1 & & \\
\hline $\mathrm{Si}$ & 0,54 & $0,38-0,77$ & 0,001 & 0,62 & $0,41-0,94$ & 0,025 \\
\hline Edad del niño en meses & 1 & $0,99-1,00$ & 0,253 & 0,99 & $0,98-1,00$ & 0,071 \\
\hline \multicolumn{7}{|l|}{ Sexo del niño } \\
\hline Masculino & 1 & & & 1 & & \\
\hline Femenino & 1,32 & $0,93-1,86$ & 0,115 & 1,37 & $0,93-2,02$ & 0,114 \\
\hline \multicolumn{7}{|l|}{ Procedencia } \\
\hline Rural & 1 & & & 1 & & \\
\hline Urbano & 2,52 & $1,39-4.59$ & 0,002 & 1,05 & $0,53-2,07$ & 0,897 \\
\hline \multicolumn{7}{|c|}{ Nivel educativo máximo alcanzado de los padres } \\
\hline Básica & 1 & & & 1 & & \\
\hline Superior & 3,31 & $2,27-4,83$ & $<0,001$ & 2,33 & $1,52-3,59$ & $<0,001$ \\
\hline \multicolumn{7}{|c|}{ Inicio de alimentación complementaria } \\
\hline Temprana & 1 & & & 1 & & \\
\hline Adecuada & 1,3 & $0,79-2,16$ & 0,305 & 1,57 & $0,86-2,90$ & 0,146 \\
\hline \multicolumn{7}{|c|}{ Antecedente de atopia por familiar directo } \\
\hline No & 1 & & & 1 & & \\
\hline $\mathrm{Si}$ & 5,02 & $3,45-7,29$ & $<0,001$ & 4,31 & $2,90-6,40$ & $<0,001$ \\
\hline \multicolumn{7}{|c|}{ Hábito de fumar en presencia del niño } \\
\hline No & 1 & & & 1 & & \\
\hline $\mathrm{Si}$ & 2,04 & $1,22-3,40$ & 0,006 & 1,76 & $0,99-3,12$ & 0,055 \\
\hline
\end{tabular}

IC 95\%: Intervalos de confianza al 95\%, OR: Odds Ratio

* Ajustado por las variables: antecedente de atopia por familiar directa, nivel educativo máximo alcanzado de los padres, hábito de fumar en presencia del niño, la edad, el sexo, inicio de alimentación complementaria y procedencia. Test de bondad de ajuste $(p=0,415)$, existe evidencia para afirmar que el modelo se ajusta a nuestros datos 
sería un gran respaldo a nuestra hipótesis y nos indicaría que el efecto protector de la LME sería en los casos más severos de DA que son los atendidos a nivel hospitalario especialmente en los de tercer nivel.

En los trabajos que evalúan la asociación entre lactancia materna y DA existen resultados variables lo cual se debería a diferencias en la forma de medición de éstas; tal es el caso de la variable presencia de lactancia materna, la cual es medida en meses de duración ${ }^{(12,14,15,19)}$ y otras, considerando diferentes puntos de corte: seis meses $(10,13,20-22)$, cuatro meses ${ }^{(11,16,17,23)}$; o cinco meses ${ }^{(24)}$; y tipo de lactancia ${ }^{(9)}$. En nuestro estudio se consideró la LME sin recibir otro tipo de alimento o suplemento. En relación al diagnóstico de DA algunos trabajos realizan dicho diagnóstico con evaluación médica o por un personal de enfermería entrenado ${ }^{(11-13,15-17,19)}$; en nuestro trabajo el diagnóstico de DA fue producto de la evaluación por especialistas dermatólogos o pediatras en consultorio externo, lo cual es una fortaleza debido a la mayor certeza diagnóstica en relación a diagnósticos basados en cuestionarios.

La mayoría de los estudios que evalúan la asociación entre lactancia materna y DA fueron estudios de cohortes; sin embargo, muchos de estos tuvieron pérdidas de seguimiento mayores al $80 \%{ }^{(13-16,21-24)}$, o no se reportaron las pérdidas (11); esto dificulta la interpretación de dichos resultados debido a que no sabemos cuál podría haber sido la evolución de los niños que no completaron dichos seguimientos. Además, el tiempo de seguimiento utilizado en estos estudios fue corto en relación al necesario para el desarrollo de la DA, el cual debería ser al menos hasta los cinco años, pues a esa edad se producen hasta el $85 \%$ de los casos ${ }^{(28)}$ y esto sólo se cumple en algunos estudios ${ }^{(12,14,15,21)}$, a diferencia del nuestro, en donde se incluyen niños evaluados hasta los siete años de edad.

Los resultados contradictorios en los estudios también podrían estar relacionados a las diferencias en los lugares de captación de la muestra, en los estudios realizados a nivel poblacional o en establecimientos de primer nivel de atención como: centros de maternidad $(11,12,14,15,24)$, centros de salud ${ }^{(16)}$, bases de datos de salud ${ }^{(17,21,23)}$, encuestas nacionales $(9,10,22)$ u otras cohortes (19) y escuelas primarias ${ }^{(20)}$ no se encuentran asociación entre lactancia materna y DA $(9,10,14,16,17,20,21,23,24)$ y de estos sólo algunos encuentran que la lactancia materna prolongada es un factor de riesgo para el desarrollo de DA ${ }^{(12,15,22)}$; mientras en los estudios realizados en consulta externa de centros hospitalarios ${ }^{(13,25)}$ encuentran que la lactancia materna está asociada a una menor frecuencia de DA, coincidiendo con lo encontrado en nuestro estudio.

El probable efecto protector de la LME sobre el desarrollo de la DA podría ser debido no solamente a la lactancia, sino a la conducta de padres con antecedentes de atopia, los cuales buscarían disminuir la exposición de sus hijos a alérgenos. En varios estudios de cohortes se encuentran que los niños que reciben lactancia materna eran hijos de madres que no fumaron durante el embarazo, tenían mayor nivel socioeconómico, tenían antecedentes de atopía, no tenían mascotas y tenían madres de mayor edad ${ }^{(11,14,15,17)}$.

El presente estudio tuvo algunas limitaciones. No fueron consideradas variables que están relacionadas a la lactancia materna como la prematuridad, el peso al nacer y sólo se evaluó el inicio de la alimentación complementaria sin considerar algún otro factor relacionado, esto debido a que se ingresaron sólo aquellas variables relacionadas a la DA referidas en estudios previos. Así también, la recolección de datos referido a los antecedentes de lactancia materna y de inicio de alimentación complementaria, dependiente de la memoria del tutor, pudo haber tenido imprecisiones en algunos casos. El diseño de muestreo utilizado (consecutivo y no aleatorio), pudo haber significado un sesgo de selección; sin embargo, se buscó controlar dicho sesgo considerando la participación de pacientes en horarios diferentes, durante aproximadamente dos años.

Por otro lado, el pareamiento por antecedente familiar de atopía hubiera sido una estrategia que hubiera ayudado en el control de este confusor, pues como se verificó en el análisis, es un factor de riesgo importante; sin embargo, no se optó por esta estrategia por la dificultad que implica, haciendo más prolongada la selección de los controles. Por ello, al realizar el análisis multivariado, buscamos controlar este efecto, como se verificó al comparar el valor crudo del OR para la lactancia materna frente al valor ajustado $(0,54$ vs 0,62$)$. A pesar de estas limitaciones, el presente estudio es una primera aproximación al tema en el Perú; además permite tener evidencia de la asociación entre DA y LME en niños menores de siete años en un hospital de tercer nivel.

En conclusión, la lactancia materna exclusiva tendría un efecto protector sobre la presencia de dermatitis atópica en niños menores de siete años, ajustado por los antecedentes de atopía de los padres, la edad y sexo del niño, el hábito de fumar en presencia del niño y el tiempo de inicio de la alimentación complementaria.

Se recomienda realizar estudios de cohortes para evaluar la asociación entre lactancia materna exclusiva y dermatitis atópica considerando otras variables no evaluadas en el presente estudio.

Contribuciónes de autoria: WMG participó en la concepción, diseño, recolección, interpretación de datos, redacción del manuscrito. JCl participó en la recolección de datos, interpretación de datos. GGV participó en el análisis de datos, interpretación de datos y redacción del manuscrito. CG participó en la concepción, diseño de proyecto, interpretación de datos, revisión crítica del contenido del manuscrito. JSF participó en la concepción, diseño del proyecto, interpretación de datos, revisión crítica del contenido del manuscrito. Todos aprobaron la versión final.

\section{Fuentes de financiamiento: Autofinanciado}

Conflicto de interes: Los autores declaran no tener conflictos de interés.

Material suplementario: Disponible en la versión electrónica de la RPMESP. 


\section{REFERENCIAS BIBLIOGRÁFICAS}

1. National Institute for Health and Care Excellence. Atopic eczema in children [Internet]. London: NICE; 2015 [citado el 15 de junio de 2015]. Disponible en: http://www.nice.org.uk/guidance/cg57

2. Deckers IAG, McLean $S$, Linssen $S$, Mommers M, van Schayck CP, Sheikh A. Investigating international time trends in the incidence and prevalence of atopic eczema 1990-2010: a systematic review of epidemiological studies. PloS One. 2012;7(7):e39803. doi: 10.1371/journal.pone.0039803

3. Solé D, Mallol J, Wandalsen GF, Aguirre V, Latin American ISAAC Phase 3 Study Group. Prevalence of Symptoms of Eczema in Latin America: Results of the International Study of Asthma and Allergies in Childhood (ISAAC) Phase 3. J Investig Allergol Clin Immunol. 2010;20(4):311-23.

4. Ellis CN, Drake LA, Prendergast MM, Abramovits W, Boguniewicz M, Daniel $\mathrm{CR}$, et al. Cost of atopic dermatitis and eczema in the United States. J Am Acad Dermatol. 2002;46(3):361-70.

5. Sanchez-Pérez J, Daudén-Tello E, Mora AM, Lara Surinyac N. Impacto de la calidad de vida relacionada con la salud en población pediátrica y adulta española con dermatitis atópica. Estudio PSEDA. Actas Dermo-Sifiliográficas. 2013;104(1):44-52. doi: 10.1016/j. ad.2012.03.008

6. Williams HC, Jburney PG, Hay RJ, Archer CB, Shipley MJ, AhunterJJ, etal. The U.K. Working Party's Diagnostic Criteria for Atopic Dermatitis. Br J Dermatol. 1994;131(3):383-96. doi: 10.1111/j.1365-2133.1994.tb08530.x.

7. Williams HC, Jburney PG, Pembroke AC, Hay RJ. The U.K. Working Party's Diagnostic Criteria for Atopic Dermatitis. III. Independent hospital validation. $\mathrm{Br}$ J Dermatol. 1994;131(3):406-16. doi: 10.1111/j.1365-2133.1994.tb08532.x.

8. Dharma C, Lefebvre DL, Tran MM, Lu Z, Lou WYW, Subbarao P, et al. Diagnosing atopic dermatitis in infancy: Questionnaire reports vs criteria-based assessment. Paediatr Perinat Epidemiol. 2018;32(6):556-67. doi: 10.1111/ ppe. 12525

9. Lee KS, Rha Y-H, Oh I-H, Choi YS, Kim Y-E, Choi SH. Does Breastfeeding Relate to Development of Atopic Dermatitis in Young Korean
Children?: Based on the Fourth and Fifth Korea National Health and Nutrition Examination Survey 20072012. Allergy Asthma Immunol Res. 2017;9(4):307-13. doi: 10.4168/ aair.2017.9.4.307.

10. Schmitz R, Atzpodien K, Schlaud M. Prevalence and risk factors of atopic diseases in German children and adolescents. PediatrAllergyImmunolOff Publ Eur Soc Pediatr Allergy Immunol. diciembre de 2012;23(8):716-23. doi: 10.1111/j.1399-3038.2012.01342.x.

11. Schoetzau A, Filipiak-Pittroff B, Franke K, Koletzko S, Von Berg A, Gruebl A, et al. Effect of exclusive breast-feeding and early solid food avoidance on the incidence of atopic dermatitis in high-risk infants at 1 year of age. Pediatr Allergy Immunol Off Publ Eur Soc Pediatr Allergy Immunol. 2002;13(4):234-42.

12. Pesonen M, Kallio MJT, Ranki A, Siimes MA. Prolonged exclusive breastfeeding is associated with increased atopic dermatitis: a prospective follow-up study of unselected healthy newborns from birth to age 20 years. Clin Exp Allergy J Br Soc Allergy Clin Immunol. 2006;36(8):1011-8. doi: 10.1111/j.1365-2222.2006.02526.x

13. Chiu C-Y, Liao S-L, Su K-W, Tsai M-H, Hua M-C, Lai S-H, et al. Exclusive or Partial Breastfeeding for 6 Months Is Associated With Reduced Milk Sensitization and Risk of Eczema in Early Childhood. Medicine (Baltimore) [Internet]. 18 de abril de 2016 [citado 24 de abril de 2018];95(15). Disponible en: https://www.ncbi.nlm.nih.gov/pmc/ articles/PMC4839855/.doi: 10.1097/ MD.0000000000003391

14. Nwaru BI, Craig LCA, Allan K, Prabhu N, Turner SW, McNeill G, et al. Breastfeeding and introduction of complementary foods during infancy in relation to the risk of asthma and atopic diseases up to 10 years. Clin Exp Allergy J Br Soc Allergy Clin Immunol. 2013;43(11):1263-73. doi: 10.1111/ cea. 12180

15. Bergmann RL, Diepgen TL, Kuss O, Bergmann KE, Kujat J, Dudenhausen JW, et al. Breastfeeding duration is a risk factor for atopic eczema. Clin Exp Allergy J Br Soc Allergy Clin Immunol. 2002;32(2):205-9.
16. Benn CS, Wohlfahrt J, Aaby P, Westergaard T, Benfeldt E, Michaelsen $\mathrm{KF}$, et al. Breastfeeding and risk of atopic dermatitis, by parental history of allergy, during the first 18 months of life. Am J Epidemiol. 2004;160(3):21723. doi: 10.1093/aje/kwh208.

17. Kull I, Wickman M, Lilja G, Nordvall SL, Pershagen G. Breast feeding and allergic diseases in infants-a prospective birth cohort study. Arch Dis Child. 2002;87(6):478-81.

18. Björkstén B, Ait-Khaled N, Innes Asher M, Clayton TO, Robertson C, ISAAC Phase Three Study Group. Global analysis of breast feeding and risk of symptoms of asthma, rhinoconjunctivitis and eczema in 6-7 year old children: ISAAC Phase Three. Allergol Immunopathol (Madr). 2011;39(6):318-25. doi: 10.1016/j. aller.2011.02.005.

19. Snijders BEP, Thijs C, Dagnelie PC, Stelma FF, Mommers M, Kummeling I, et al. Breast-feeding duration and infant atopic manifestations, by maternal allergic status, in the first 2 years of life (KOALA study). J Pediatr. 2007;151(4):347-51, 351.e1-2. doi: 10.1016/j.jpeds.2007.03.022.

20. Morales-Romero CJ, Bedolla-Barajas M, López-Vargas L, Romero-Velarde CE. Prevalence of allergic diseases and their association with breastfeeding and initiation of complementary feeding in school-age children of Ciudad Guzmán, Mexico. Arch Argent Pediatr. 2015;113(4):324-30.doi: 10.5546/ aap.2015.324.

21. Wang J, Ramette A, Jurca M, Goutaki M, Beardsmore CS, Kuehni CE. Association between breastfeeding and eczema during childhood and adolescence: A cohort study. PloS One. 2017;12(9):e0185066. doi: 10.1371/ journal.pone.0185066.

22. Ito J, Fujiwara T. Breastfeeding and risk of atopic dermatitis up to the age 42 months: a birth cohort study in Japan. Ann Epidemiol. 2014;24(4):267-72. doi: 10.1016/j.annepidem.2013.11.007.

23. Ludvigsson JF, Mostrom M, Ludvigsson J, Duchen K. Exclusive breastfeeding and risk of atopic dermatitis in some 8300 infants. Pediatr Allergy Immunol Off Publ Eur Soc Pediatr Allergy Immunol. 2005;16(3):201-8. doi: 10.1111/j.1399-3038.2005.00257.x. 
24. Parazzini F, Cipriani S, Zinetti C, Chatenoud L, Frigerio L, Amuso G, et al. Perinatal factors and the risk of atopic dermatitis: a cohort study. Pediatr Allergy Immunol Off Publ Eur Soc Pediatr Allergy Immunol. 2014;25(1):43-50. doi: 10.1111/ pai. 12165 .

25. Goicochea VWA, Hidalgo TCM, Hérnandez DH, Centeno HJ. Asociación entre prematuridad, bajo peso al nacer y lactancia materna exclusiva con rinitis alérgica, en niños de 2 a 7 años que acuden al Hospital Nacional Cayetano Heredia,
Perú. Bol Méd Hosp Infant México. 2010;67(4):315-26.

26. Perú, Instituto Nacional de Estadística e Informática. Perú: Encuesta Demográfica y de Salud Familiar 2014 - Nacional y Departamental [Internet]. Lima: INEI 2015 [citado 30 de abril de 2018]. Disponible en: https://www.inei.gob.pe/ media/MenuRecursivo/publicaciones_ digitales/Est/Lib1211/index.html

27. Morales Suárez-Varela M, García-Marcos L, Kogan MD, Llopis González A, Martínez Gimeno A, Aguinaga Ontoso I, et al. Parents' smoking habit and prevalence of atopic eczema in 6-7 and
13-14 year-old schoolchildren in Spain. ISAAC phase III. Allergol Immunopathol (Madr). 2008;36(6):336-42.

28. Hurwitz, Paller AS, Mancini AJ. Dermatología pediátrica: texto de enfermedades de la piel en niños y adolescentes. Madrid: Marbán; 2014.

Correspondencia: Winston Maldonado-Gómez Dirección: Calle San José 125 Oficina 225,

Chiclayo. Lambayeque, Perú.

Teléfono: (+511) 978071294

Correo electrónico: winstonmaldonado16@ gmail.com

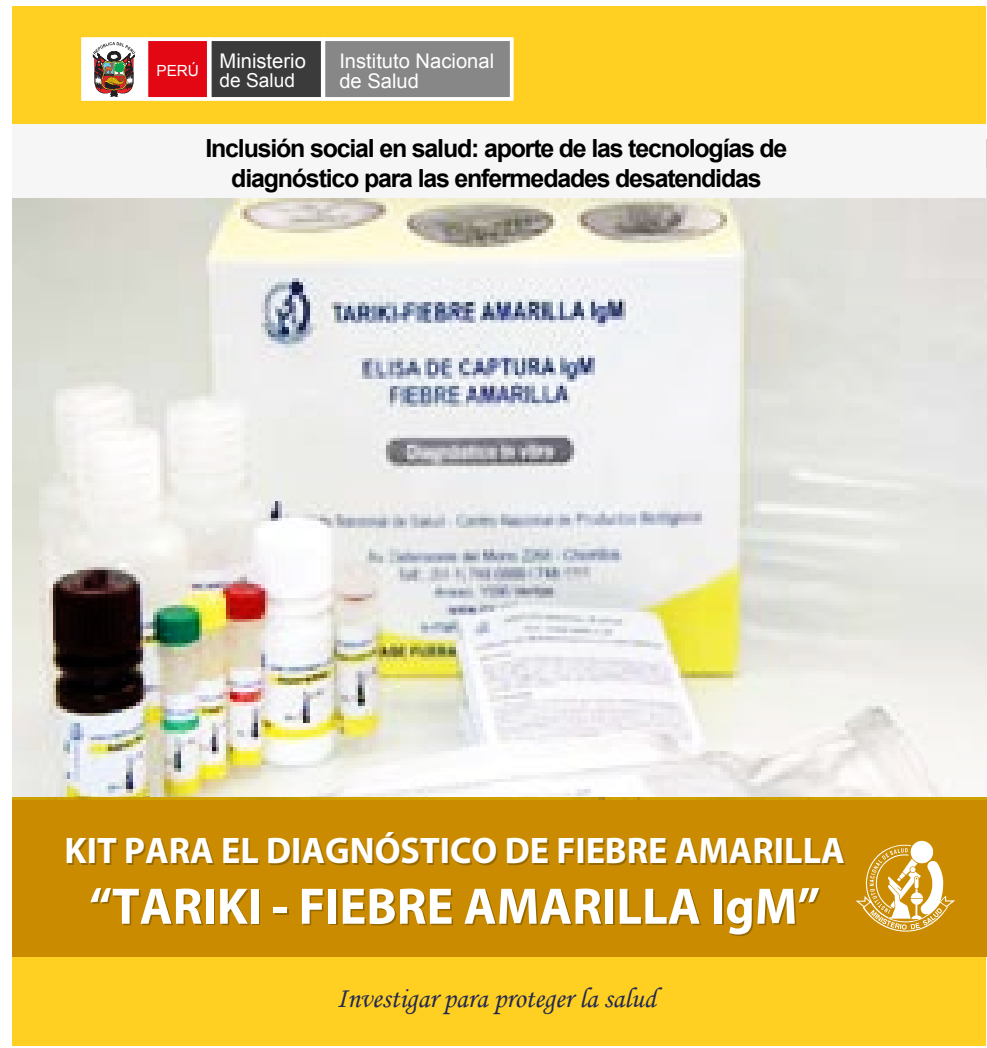

